\title{
Expression of ZIC Genes in the Development of the Chick Inner Ear and Nervous System
}

\author{
Stephen J. Warner, ${ }^{1}$ Mary R. Hutson, ${ }^{2}$ Seung-Ha Oh, ${ }^{1,3}$ Lisa M. Gerlach-Bank, ${ }^{2,4}$ Margaret I. Lomax, ${ }^{1,2}$ and \\ Kate F. Barald ${ }^{2,4 *}$
}

\begin{abstract}
ZIC genes, vertebrate homologues of the Drosophila pair-rule gene odd-paired (opa), function in embryonic pattern formation, in the early stages of central nervous system neurogenesis and in cerebellar maturation. Mouse Zic genes are expressed in restricted, and in some cases overlapping, patterns during development, particularly in the central and peripheral nervous systems. We identified chick ZIC2 in a differential display analysis of the auditory system designed to find genes up-regulated after noise trauma. In this study, we examined the expression of chick ZIC1, ZIC2, and ZIC3 by in situ hybridization in normal inner ear development and in the tissues that influence its development, including the hindbrain, the neural crest, and the periotic mesenchyme. Between Hamburger and Hamilton stages 13 and 24, all three ZIC genes were found in the dorsal periotic mesenchyme adjacent to the developing inner ear. ZIC1 mRNA was expressed in the otocyst epithelium between stages 12 and 24, in some sensory tissue, as well as in a striped pattern in the floorplate of the hindbrain that appears to be complementary to that of Chordin, a gene known to regulate ZIC expression in frogs. Chick ZIC genes are also expressed in the neuroectoderm, paraxial mesenchyme, brain, spinal cord, neural crest, and/or the overlying ectoderm as well as the limb buds. In general, ZIC1 and ZIC2 expression patterns overlapped, although ZIC2 expression was less robust; ZIC3 expression was minimal. These observations suggest that ZIC genes, in addition to their known roles in brain development, may play an important role in the development of the chick inner ear. Developmental Dynamics 226:702-712, 2003.
\end{abstract} ๑) 2003 Wiley-Liss, Inc.

Key words: zinc finger protein; ear development; hindbrain; neural crest; periotic mesenchyme; chondrocytes

Received 29 April 2002; Accepted 27 November 2002

\section{INTRODUCTION}

The inner ear develops from an epithelial thickening, the otic placode that forms adjacent to rhombomeres 5 and 6 of the developing hindbrain. The development of the inner ear is strongly influenced by molecular signals from the hindbrain, the periotic mesenchyme
(POM), and the notochord, as well as the neural crest (NC; Fritzsch et al., 1997), all of which act as sources of molecular inductive cues. As in all vertebrates, the chick otic placode gives rise to the majority of cell lineages in the inner ear's complex three-dimensional structure (reviewed in Fritzsch et al.,
1997; Torres and Giraldez, 1998; Fekete, 1999).

Chick inner ear organogenesis provides an excellent model for studying the well-conserved patterning roles of morphogen/antagonist interactions during development (reviewed in Giudice, 2001). The molecules involved in early inner ear

\footnotetext{
${ }^{1}$ Kresge Hearing Research Institute, Department of Otolaryngology/Head-Neck Surgery, University of Michigan Medical School, Ann Arbor, Michigan

${ }^{2}$ Department of Cell and Developmental Biology, University of Michigan Medical School, Ann Arbor, Michigan

${ }^{3}$ Department of Otolaryngology-Head and Neck Surgery, Seoul National University College of Medicine, Chongno-ku, Seoul, Korea

${ }^{4}$ Cell and Molecular Biology Program, University of Michigan, Ann Arbor, Michigan

Grant sponsor: National Institutes of Health; Grant numbers: DC04184; DC05939; DC02492; Grant sponsor: National Science Foundation; Grant number: IBN 9906424; Grant sponsor: NIH Training Grants; Grant numbers: NIDCD T32 DC00011; GM07315-26.

Dr. Hutson's present address is Department of Pediatrics, Duke University Medical School, Durham, NC.

*Correspondence to: Kate F. Barald, University of Michigan Medical School, Department of Cell and Developmental Biology, Box 0616, 1150 W. Medical Center Drive, Ann Arbor, MI 48109-0616. E-mail: kfbarald@umich.edu
}

DOI 10.1002/dvdy.10262 
axial specification, the three-dimensional organogenesis of semicircular canals in three different planes, and sensory development within specialized auditory and vestibular regions include members of the transforming growth factor $\beta$ (TGF $\beta$ ) superfamily. The bone morphogenetic proteins (BMPs) and their molecular antagonists such as Chordin, Noggin, and DAN are found to be critical for inner ear three-dimensional patterning and sensory cell formation (Chang et al., 1999; Gerlach et al., 2000; Gerlach-Bank et al., 2002).

We have demonstrated that perturbing BMP4/Noggin or BMP4/DAN signaling also affected the early patterning of inner ear sensory organs and the structures that enclose them. For example, delivering exogenous Noggin to the developing otocyst by implanting beads bearing Chinese hamster ovary $(\mathrm{CHO})$ cells that produce Noggin (Gerlach et al., 2000) resulted in abnormal phenotypes affecting primarily the semicircular canals (SCC) and their enclosed sensory tissues (Gerlach et al., 2000). Similar results were found when a Noggin-producing retrovirus was introduced (Chang et al., 1999). We also produced similar inhibitory effects on SCC morphogenesis with exogenous DAN, a second BMP4 antagonist (Gerlach-Bank et al., 2002).

BMP4 and Chordin constitute another molecular morphogen/antagonist pair. Chordin is also involved in neurogenesis in the chick, in opposition to the effects of BMP4 (Streit et al., 1998; Streit and Stern, 1999; Larrain et al., 2000). The BMP4/Chordin balance, in turn, is upstream of and regulates several genes, most significantly, the ZIC genes (Aruga et al., 1996; Nagai et al., 1997). In Xenopus, up-regulation of Chordin is known to up-regulate $\mathrm{ZICl}$, which was isolated in a screen for neural inducers in the frog, i.e., genes activated by Chordin and blocked by BMP4 (Mizuseki et al., 1998). In contrast, BMP4, as a molecular antagonist of Chordin, down-regulates $\mathrm{ZICl}$.

Aruga et al. (1994, 1996) first identified the mouse Zic genes as the vertebrate homologues of Drosophila opa and studied their expression in early mouse embryos (Nagai et al., 1997). They reported expression in dorsal neural ectoderm, paraxial mesenchyme, brain, spinal cord, and epidermis; the marginal zone of the neural retina and distal regions of the developing limb. Mouse Zic 7 is expressed in cerebellar granule cells throughout prenatal and postnatal development (Aruga et al., 1994) and is also considered to be a molecular marker for granule cells in the dorsal cochlear nucleus (Ivanova and Yuasa, 1998). Targeted inactivation of mouse Zicl affected brain development (Aruga et al., 1998), producing an effect that mimics holoprosencephaly (HPE). Reduced expression of mouse Zic2 results in neurulation delay, causing both HPE and spina bifida (Nagai et al., 2000). Mutations in ZIC2 also cause HPE in humans (Brown et al., 1998).

In earlier studies, we identified a partial chick CDNA for ZIC2 in a differential display experiment designed to find genes whose expression is elevated after noise trauma in the chicken (Gong et al., 1996). Chick inner ear sensory epithelium, unlike that of mammals, is capable to some extent of regeneration (Stone and Rubel, 2000). Thus genes that are upor down-regulated after noise exposure in the chick inner ear might be candidates for involvement in regeneration. Because genes that are candidates for involvement in sensory epithelium regeneration are probably also important in development, we examined the expression patterns of chick ZIC genes during the process of normal inner ear development and in the tissues that are known to affect this development. We discuss the possible role of the ZIC transcription factors in both inner ear development and regeneration.

\section{RESULTS AND DISCUSSION ZIC1, ZIC2, and ZIC3 Expression in the Dorsal Neural Tube, Hindbrain, and Neural Crest}

We used whole-mount in situ hybridization followed by cryosectioning or direct labelling of sectioned material of chick embryos between Hamburger and Hamilton (1951) stages 10 to 32 to determine the overall pattern of ZIC gene expression in early em- bryos and to assess the potential role of ZIC genes in early stages of chick nervous system development. Expression of ZIC genes was detected in the neural tube (NT) and hindbrain (HB) as early as stages 10 (not shown) and 12 (Fig. 1A). ZICl consistently showed the highest expression and ZIC 3 the lowest in all regions at all stages (Figs. 1, 2). Neural tube expression is limited to the dorsomedial region until stage 14. At stage 18 (Fig. 1C), some medial NT expression in the vicinity of the otocyst (OC) is seen, and the periotic mesenchyme also begins to express $\mathrm{ZICl}$ at high levels between stages 21 (Fig. 1D) and 32 (Fig. 1H). Between stages 21 (Fig. 1D) and 23 (Fig. 1E), the luminal half of the HB epithelium expresses a high level of $Z I C l$, and a sharp boundary is established between the luminal half of the lateral epithelium and the half of the NT epithelium that borders the POM/otocyst. By stage 24 (Fig. 1F), the level of expression of ZIC1 in the periotic mesenchyme, particularly around the endolymphatic duct and sac (endolymphatic apparatus, labelled EA in Fig. 1F) and lateral to the otic epithelium is very high. At stage 32 (Fig. $1 \mathrm{H}$ ), a gradient of $\mathrm{ZICl}$ expression is seen from medial (high) to lateral (low).

We compared the expression patterns of all three ZIC genes in the neural tube and trunk neural crest at stage 23 (Fig. 2). Both $\mathrm{ZICl}$ (Fig. 2A) and ZIC2 (Fig. 2B) have distinct but overlapping expression patterns in the dorsal neural tube and the surrounding mesenchyme. Both genes were expressed in the roof plate (RP) of the neural tube; however, the region of $\mathrm{ZICl}$ expression was more extensive than that of $Z I C 2$ in the RP. In contrast to $Z I C 1$, low levels of ZIC2 mRNA were also found medially in the neural tube (Fig. 2B) in two dorsal to ventral stripes (the right stripe is indicated by an asterisk). The regions with high levels of $Z I C 1$ and $Z I C 2$ expression in the surrounding paraxial mesenchyme coincided with the three NC migratory pathways, suggesting that these ZICexpressing cells are NC cells or their derivatives. The strongest expression of $\mathrm{ZICl}$ and $\mathrm{ZIC} 2$ was found in the migrating NC in the dorsolateral and ventromedial pathways, shown diverging around the DRGs in these photographs (Fig. 2A,B). ZIC2-express- 


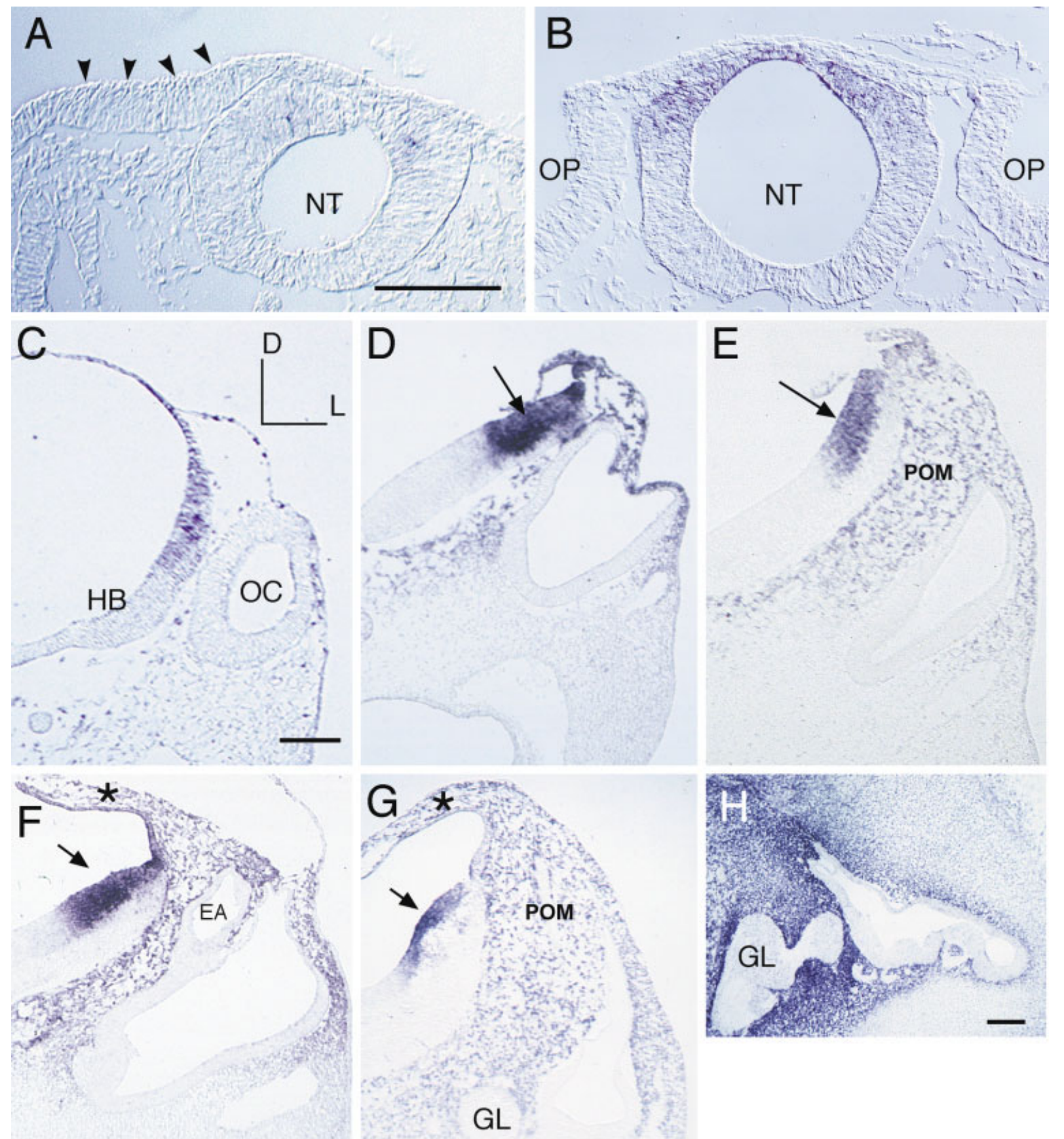

Fig. 1.
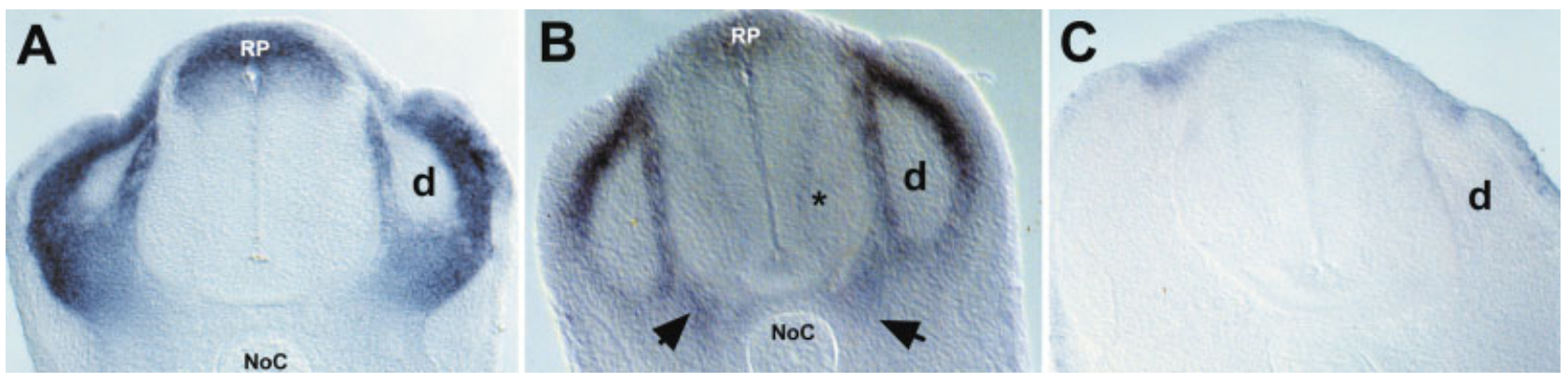

Fig. 2. 
TABLE 1. Summary of ZIC1, ZIC2, and ZIC3 Expression During Early Inner Ear Development

\begin{tabular}{|c|c|c|c|c|c|c|}
\hline Gene & Stage & $\begin{array}{l}\text { Neural } \\
\text { tube }\end{array}$ & $\begin{array}{l}\text { Limb } \\
\text { buds }\end{array}$ & Hindbrain & $\begin{array}{l}\text { Periotic } \\
\text { mesenchyme }\end{array}$ & $\begin{array}{l}\text { Presensory/sensory } \\
\text { epithelium }\end{array}$ \\
\hline \multirow[t]{5}{*}{$Z I C 1$} & 10 & + & & + & & - \\
\hline & 13 & + & & + & & + \\
\hline & 18 & + & + & + & + & + \\
\hline & 24 & + & + & $+^{a}$ & + & + \\
\hline & 32 & & & + & + & + \\
\hline \multirow[t]{3}{*}{ ZIC2 } & 13 & + & & + & & $?$ \\
\hline & 18 & + & + & + & + & $?$ \\
\hline & 24 & + & + & + & + & $?$ \\
\hline \multirow[t]{3}{*}{ ZIC3 } & 13 & + & + & + & & + \\
\hline & 18 & + & + & + & + & + \\
\hline & 24 & + & + & + & + & $?$ \\
\hline
\end{tabular}

aStriped pattern (see Figs. 3,4 ) detected at this stage as in the stage 23 embryos illustrated.

ing cells extended more ventrally than ZIC 1-expressing cells and included cells just above the notochord (compare Fig. 2A with B, arrowheads). Although ZIC 1, ZIC2, and ZIC3 share similar patterns of expression in other areas (Table 1), ZIC3 was found to be minimally expressed in the NC and largely confined to the overlying dorsal ectoderm (Fig. 2C). These expression patterns for chick ZICl and ZIC2 in neural tube and chick ZIC3 in the dorsal ectoderm are consistent with previous studies in the mouse (Nagai ef al., 1997) and in Xenopus (Nakata et al., 1998).

\section{ZICl and Chordin Expression in} the Neural Tube

The ZICl expression pattern in the floorplate of the hindbrain (Fig. 3C) and midbrain (Fig. 3D) is interdigitated with that of the BMP4 antagonist Chordin, a gene hypothesized to regulate $\mathrm{ZIC}$ gene expression, at least in the frog (Nakata et al., 1997, 1998). We performed double in situ hybridization experiments with probes for ZICl and Chordin mRNA (Fig. 3). In an otic section (Fig. 3A), $\mathrm{ZICl}$ expression is again seen in the neural epithelium of the dorsolateral hindbrain on the luminal side (as in Fig. 1E-G). In a more rostral section (Fig. 3B), this pattern is recapitulated. Strong ZIC 7 labeling is also found in the lateral hindbrain (as seen in Fig. $3 \mathrm{C}$ bottom; see also Fig. 1C-F) in the roofplate (arrows in Fig. 3D) and in the somatic mesoderm outside the hindbrain (Fig. 3E). More importantly, interdigitated Chordin/ZIC labeling is found in the floorplate of the hindbrain (Fig. 3C) and midbrain (Fig. 3D). Because Chordin is hypothesized to up-regulate $Z I C l$, the clear separation of the two expression patterns (Figs. 3C,D, 4D) may define

Fig. 1. $\mathrm{ZICl}$ expression in early developing chick inner ear from stage 12 to stage 32 . In situ hybridization was performed on frozen transverse cross-sections in the region of the hindbrain (HB) and otic epithelium (see Experimental Procedures section). The stages illustrated are stage 12 (A), stage 14 (B), stage 18 (C), stage 21 (D), stage $23(E)$, stage $24(F)$, stage 26 (G), and stage 32 (H). Stage 12 (A) and $(B)$ stage $14 \mathrm{ZICl}$ expression is found in two dorsal bilateral cell foci in the neural tube (NT; compare also with Fig. 5A). In $A$, the otic placode, which did not express $Z I C l$, is indicated by arrowheads. In $\mathrm{B}$, the stage 14 embryo, no ZICl expression is seen in this particular section of the otic pit, but compare this section with that of Figure 4A,B where a small focus of cells at the tops of the pit expresses ZICl. By stage $18(\mathrm{C})$, mesenchymal cells dorsal to the otocyst (OC) have begun to express $\mathrm{ZICl}$. As the otocyst matures (C-H), the number of ZIC I-positive cells in the periotic mesenchyme (POM) increases, especially dorsal and medial to the otocyst and in the epithelium dorsal to the neural tube (asterisks in F,G; D-F). In these sections, the only ZICl hindbrain signal is found in the dorsolateral portion of the HB (indicated by arrows in $D-G$ ), but in a narrow more rostral section (Fig. $4 D$ ), $Z I C l$ is found in a striped pattern throughout the $\mathrm{HB}$ and in the medial otic epithelium. D, dorsal; L, lateral; EA, endolymphatic apparatus; GL, statoacoustic ganglion. Scale bars = $100 \mu \mathrm{m}$ in A (applies to $A, B$ ), in $C$ (applies to $C-E$ ), in $F$ (applies to $F, G$ ), in $H$.

Fig. 2. In situ hybridization analysis of ZIC gene expression in the neural tube and neural crest. Whole-mount in situ hybridization with $Z I C I, Z I C 2$, and ZIC3 probes was performed in stage 23 chick embryos. Embryos were then cryoprotected and sectioned through the neural tube and dorsal root ganglion (DRG; d). These whole-mount in situ hybridization studies demonstrated the specificity of the probes and showed that the three ZIC genes have different expression patterns in this region. A: The roofplate (RP) of the hindbrain is heavily labeled by a probe for ZICl. Two neural crest streams, one dorsolateral to the DRG (d) and one ventromedial, are also prominently labeled but do not reach the vicinity of the notochord (NoC), whose dorsal half can be seen in the midline of the photograph. B: ZIC2 labels a smaller area of the RP, two streams of cells in the middle of each side of the NT (the right stream is indicated by an asterisk), and both neural crest streams on either side of the DRGs (d). Note that the medial NC stream approaches the NoC (arrowheads) more closely than does the area of ZICl-expressing cells. C: Only the dorsal epithelium expresses ZIC3. There is no expression in the NC or hindbrain roofplate. Original magnifications, $\times 10$. Dorsal is to the top in these photographs. 

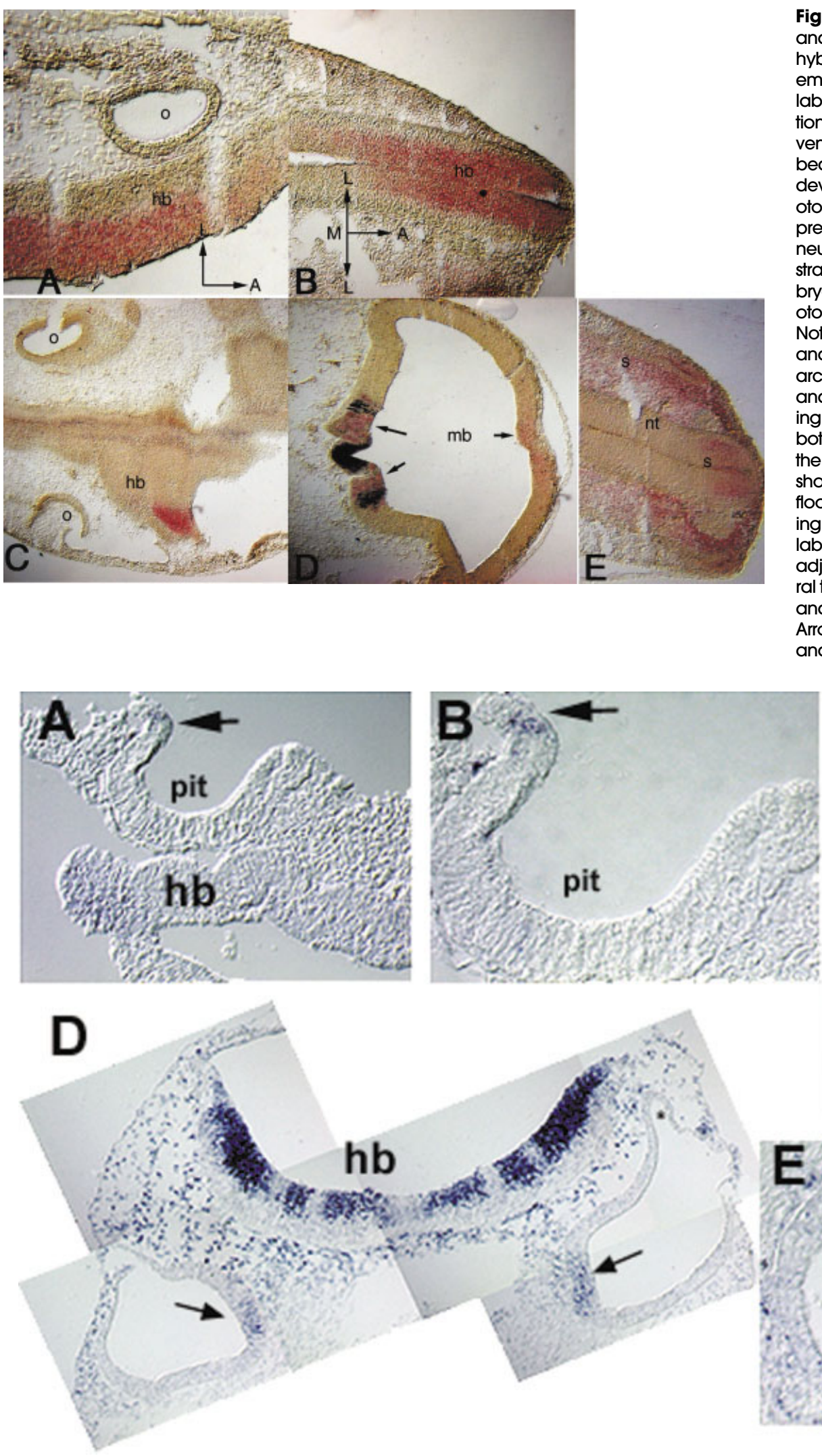

Fig. 4. $\mathrm{ZICl}$ expression in the otic pit, otic epithelium, and hindbrain at stages 12,18 , and 23. A: Stage 12 . Transverse section through the otic pit (pit) and hindbrain (hb) of a stage 12 embryo showing $\mathrm{ZICl}$ expression (arrow) at the edge of the otic pit. B: Stage 12. Higher magnification of A to illustrate the small number of labeled cells (arrow). C: Stage 18. Transverse section through the otocyst of a stage 18 embryo. ZIC 1 is expressed in the medial wall of the otic epithelium (arrow). D: Stage 23. Transverse section through the developing inner ear of a stage 23 embryo through the limited region of otic epithelial ZICl expression in the medial walls (arrows). $Z I C l$ is also expressed in the periotic mesenchyme and in a striped pattern within the floorplate of the hb. Note the emerging endolymphatic duct from the dorsolateral area of the otocyst (asterisk on the right otocyst). E: ZICl expression in the crista is seen in the sensory epithelium. Original magnifications, $\times 25$ in A-E.
Fig. 3. Complementary expression of $\mathrm{ZICl}$ and Chordin in the floorplate. Double in situ hybridization was performed on stage 23 chick embryos with Chordin and ZICl probes. ZICI labeling is red, Chordin is deep purple. Sections (nonserial; $14 \mu \mathrm{m}$ ) proceed from dorsal to ventral through the embryo, which was emdeveloping hindbrain (hb), adjacent to the cyst (0), which in this section does not ex(a) strating expression of $Z 1 C l$ in the stage 23 . otocysts and the floorplate of the hindbrain. Note that the Chordin stripes (purple black) and the paler interdigitated red $\mathrm{ZICl}$ stripes are arched, following the rhombomeric pattern and that a large area of prominent ZICl labeling can also be seen laterally, which is at the bottom of the photograph. D: Sections through the midbrain $(\mathrm{mb})$ floorplate of the embryo showing three stripes of Chordin labeling in the floorplate as well as striped interdigitated labeling of $Z I C l$. Arrows indicate the sites of $Z I C l$ labeling. $\mathrm{E}: \mathrm{ZICl}$ expression is maintained in the adjacent somitic mesenchyme and in the neural tube. A, anterior; $M$, medial; L, lateral. Dorsal and/or anterior are to the right in all figures. Arrows in $\mathrm{D}$ indicate stripes of $\mathrm{ZICl}$ in the floorand roofplate. Original magnifications, $\times 10$. bedded on its back. A: $\mathrm{ZICl}$ expression in the bryo. C: A more ventral section through the
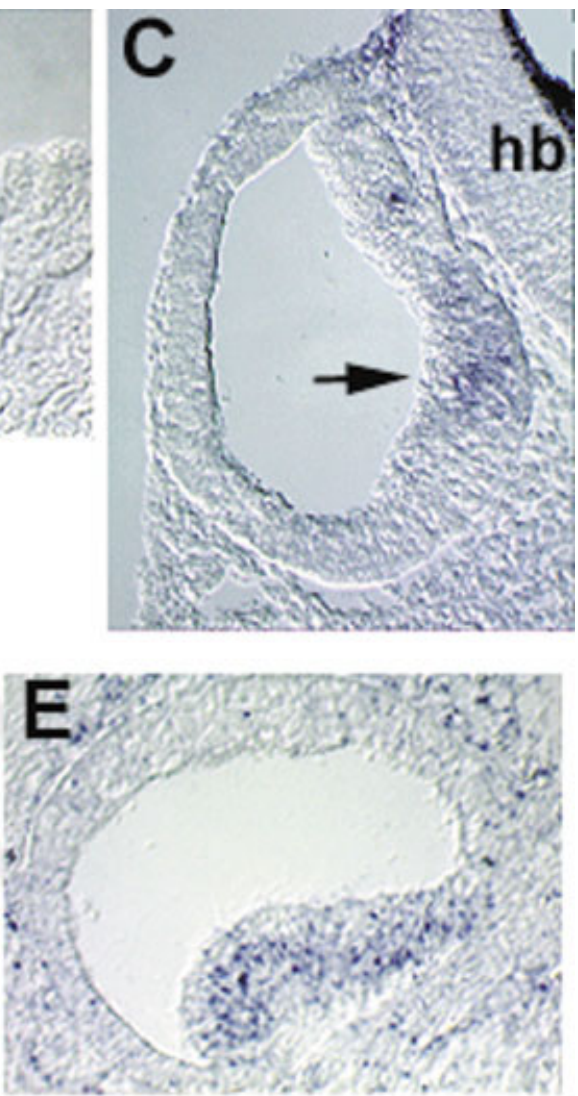
local boundaries critical for this regulation. It is not yet known whether ZIC 7 up-regulation occurs at specific times in development, because ZICI expression in the floorplate appears to be up-regulated at certain stages in development and then to wane (data not shown).

\section{ZICl Expression in the Otic Pit, Otic Epithelium, and ZIC Gene Expression in the Periotic Mesenchyme}

We examined ZIC gene expression during inner ear development by in situ hybridization on both coronal and transverse sections through the otocyst, POM, and hindbrain of early chick embryos, and also on horizontal sections through the basilar papillae and vestibular organs from embryonic day 4 (E4) to hatching, and in posthatch day 7 (P7) chicks. At most stages, $\mathrm{ZICl}$ expression was consistently higher than $Z I C 2$, and $\mathrm{ZIC} 3$ was minimal. At stages 10 and 12, we detected ZICI mRNA in a region of the hindbrain adjacent to the otic placode but not in the placode itself (refer to Fig. 1A; the placode is indicated by arrowheads). At stage $12, \mathrm{ZICl}$ is expressed at the edges of the otic pit in epithelial cells that may be involved in closing the otic pit to form the otocyst (Fig. 4A,B) or that mark the boundary between the otic pit and the surrounding neuroectoderm. ZICl mRNA is detectable in the otic epithelium by in situ hybridization at stages 12, 18 (Fig. 4A-C), 23 (Fig. 5B), and 32 (Fig. 6; summarized in Table 1). At stages 18 and $23, Z I C 7$ is expressed in the medial wall of the otocyst (Fig. 4C,D, arrows; Figs. 5B, 6B). In the narrow rostral to caudal area in which we found $\mathrm{ZICl}$ medial otocyst expression at stage 23, we also found it in a narrowly defined striped pattern in the hindbrain (Fig. 4D). Neither this striped hindbrain pattern nor the medial otocyst expression is seen in most sections of the HB and otocyst that are more rostral or more caudal (compare Fig. 4D with 1D-F). A similar narrowly defined striped pattern is seen in the localization of EphA4 tyrosine kinase receptor, expressed in a limited area in rhombomere 5 at stage 25 (Cramer et al., 2000).

\section{Coexpression of $\mathrm{ZICl}$ and the transcription factor Pax2}

Pax2 is one of the earliest genes/ proteins expressed in a regionalized pattern in the developing inner ear (Herbrand et al., 1998; Hutson et al., 1999). We examined the relationship of ZIC gene expression to that of this early gene. At stage 12, when most of the otic epithelium expresses Pax2 protein, ZICl is expressed only in a small number of cells in the neural tube (Fig. 5A). However, at stage 24, ZICl is also found coexpressed with Pax2 protein in the otic epithelium (Fig. 5B). In earlier studies, we demonstrated that the first region of the chick otocyst to express and maintain Pax2 is the dorsomedially emerging endolymphatic duct/ sac (ED/ES). When the position of the emerging ED was altered with respect to its environment, either by 180 degrees otocyst rotations about the anteroposterior axis, which placed the ED/ES on the opposite side of the otocyst with respect to the HB, or transplantation of the otocyst into the hindbrain cavity, Pax2 expression remained high in the ED. However, in the rest of the otic epithelium, Pax2 expression reverted to a pattern in which it was higher on the hindbrain side, as in the normal expression pattern. Postrotation, Pax2 expression was up-regulated on the side of the otocyst closest to the HB and down-regulated on the side that was turned away from the $\mathrm{HB}$, indicating that Pax2 expression is plastic and influenced by the environment (Hutson et al., 1999).

\section{Some Sensory Epithelial Cells Express $\mathrm{ZICl}$}

In the mature stage 32 inner ear, when the membranous labyrinth is morphologically distinct, $\mathrm{ZIC} 1$ is also expressed in both the sensory and supporting cells of the sensory epithelium in the superior crista ampullaris (Figs. 4E, 6E), in the cochlear duct epithelium of the basilar papilla (Fig. 6C) and in the sacculus, although whether this staining is indeed in the macula will require additional double labelling with, for example, hair cell-specific antibodies (Fig. 6A). The nonsensory epithe- lium of the endolymphatic duct and sac also expresses ZICl (Fig. 6D).

\section{Otic Expression of ZIC2 Is Largely Confined to the Periotic Mesenchyme}

Between stages 13 and 18, expression of $\mathrm{ZICl}, \mathrm{ZIC2}$, and $\mathrm{ZIC} 3$ in the POM was limited to the POM surrounding the dorsal half of the otocyst between the hindbrain and the otocyst, in a location consistent with that of the ZIC 7-positive migrating NC cells seen in Figure 2. In stage 21-24 embryos, the same pattern of expression in the POM surrounding the dorsal half of the otocyst was found for all three ZIC genes, illustrated for ZIC2 in the stage $22 \mathrm{em}$ bryo in Figure 7 (also compare the coronal sections in this figure with the transverse sections in Fig. 1D-G), where the POM labelling pattern is the same. These data are also summarized in Table 1. Stage 18-24 chick embryos also showed high levels of $Z I C 1, Z I C 2$, and ZIC3 mRNA in the limb buds (not shown).

\section{ZIC Expression During Later Stages of Inner Ear Development}

By E4, changes in the shape of the otocyst are evident (Bissonnette and Fekete, 1996), the most distinctive being the formation of a hollow tube on the medial surface that will become the endolymphatic duct and eventually produce the endolymphatic sac at its tip (also called the EA). We examined both $\mathrm{ZICl}$ and ZIC2 expression in adjacent sections at stages between E4 to P7. ZICl expression at this time is largely confined to the periotic mesenchyme (Fig. 8A) between the otocyst and the HB. By E7, cartilage is beginning to condense around the inner ear; $\mathrm{ZICl}$ expression is high in the condensing cartilage (Ca), particularly around the statoacoustic ganglion. ZIC2 expression was coincident in the POM with that of $\mathrm{ZICl}$ at most stages but was less robust (see Fig. $8 C, D$, where adjacent sections were hybridized with $Z I C I$ and $Z I C 2$, respectively). By E7 (stage 32), both the cochlea with its sensory epithe- 
lium (basilar papilla) and the vestibular organs have been formed. ZIC 7 was expressed at a high level in the POM between E4 and E7, but ex-

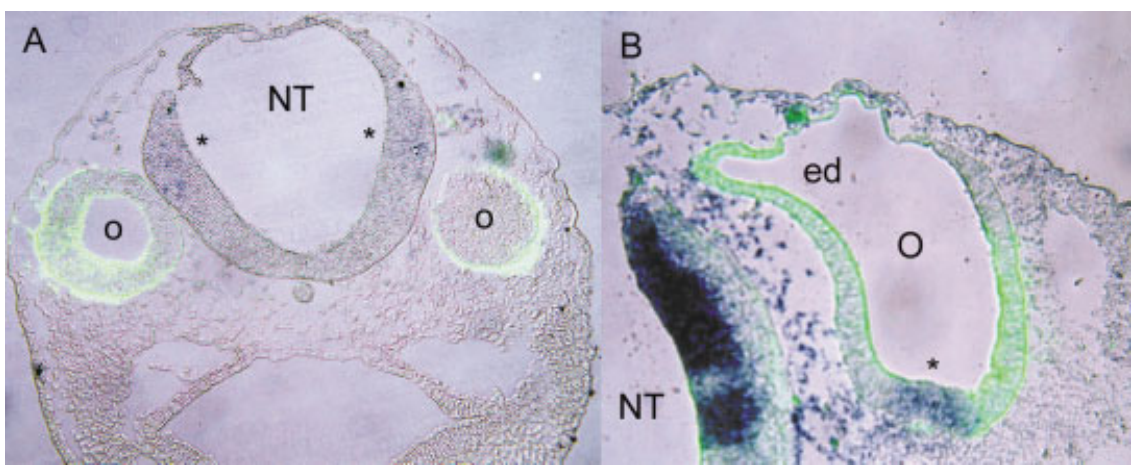

Fig. 5. ZICl in situ hybridization (dark blue) and antibody to Pax2 (green) labeling in the developing inner ear and neural tube of stage 14 (A) and stage 23 (B) embryos. A: Pax2 is confined to the otocysts in the stage 14 embryo in this particular section of the stage 14 embryo. B: Pax2 and ZICl are expressed in both the lateral neural tube and the otocyst in this section of the stage 23 embryo. ed, endolymphatic duct; $O, O$, otocyst; NT, neural tube. Original magnifications, $\times 10$ in $A, \times 25$ in $B$.

pression decreased and became more restricted after that time.

By E7 and later, part of the POM is committed to becoming cartilage,

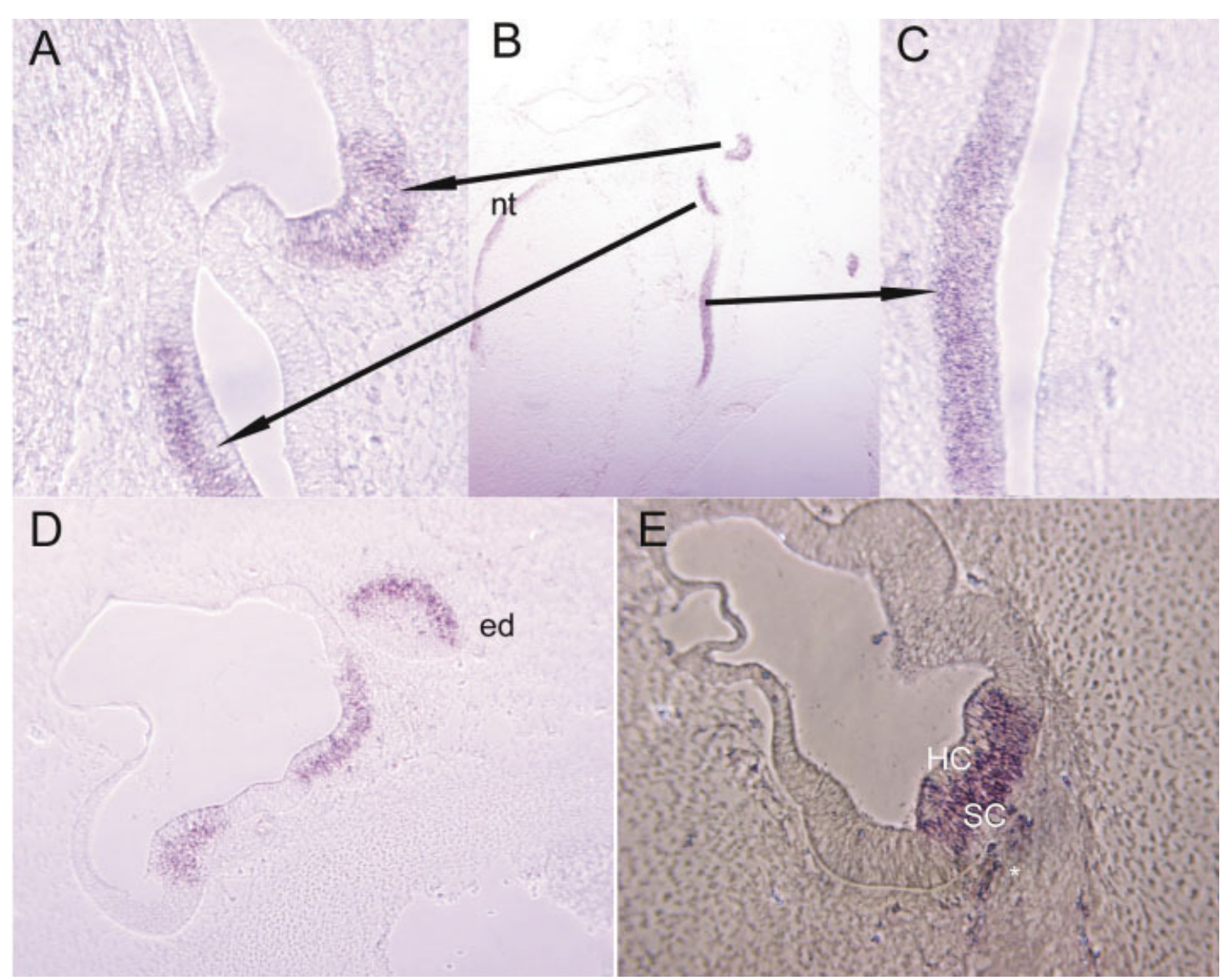

Fig. 6. B: A stage 32 (embryonic day 7) chick inner ear sectioned transversely and labeled with a probe for $\mathrm{ZICl}$ is seen at low power $(\times 4)$. Arrows connect the low-power view, provided to give orientation and relationships of the labeled areas, with the higher power views ( $\times 25$ ) seen in A and C. A: ZICl is expressed in the sacculus. The basilar papilla of the cochlear duct also expresses ZICl (C), as do the endolymphatic duct (ed) and sac (D). E: A section of another embryo through the crista ampullaris. Both hair cells (HC) and supporting cells (SC) express ZICl. Original magnifications, $\times 25$ in D,E. 


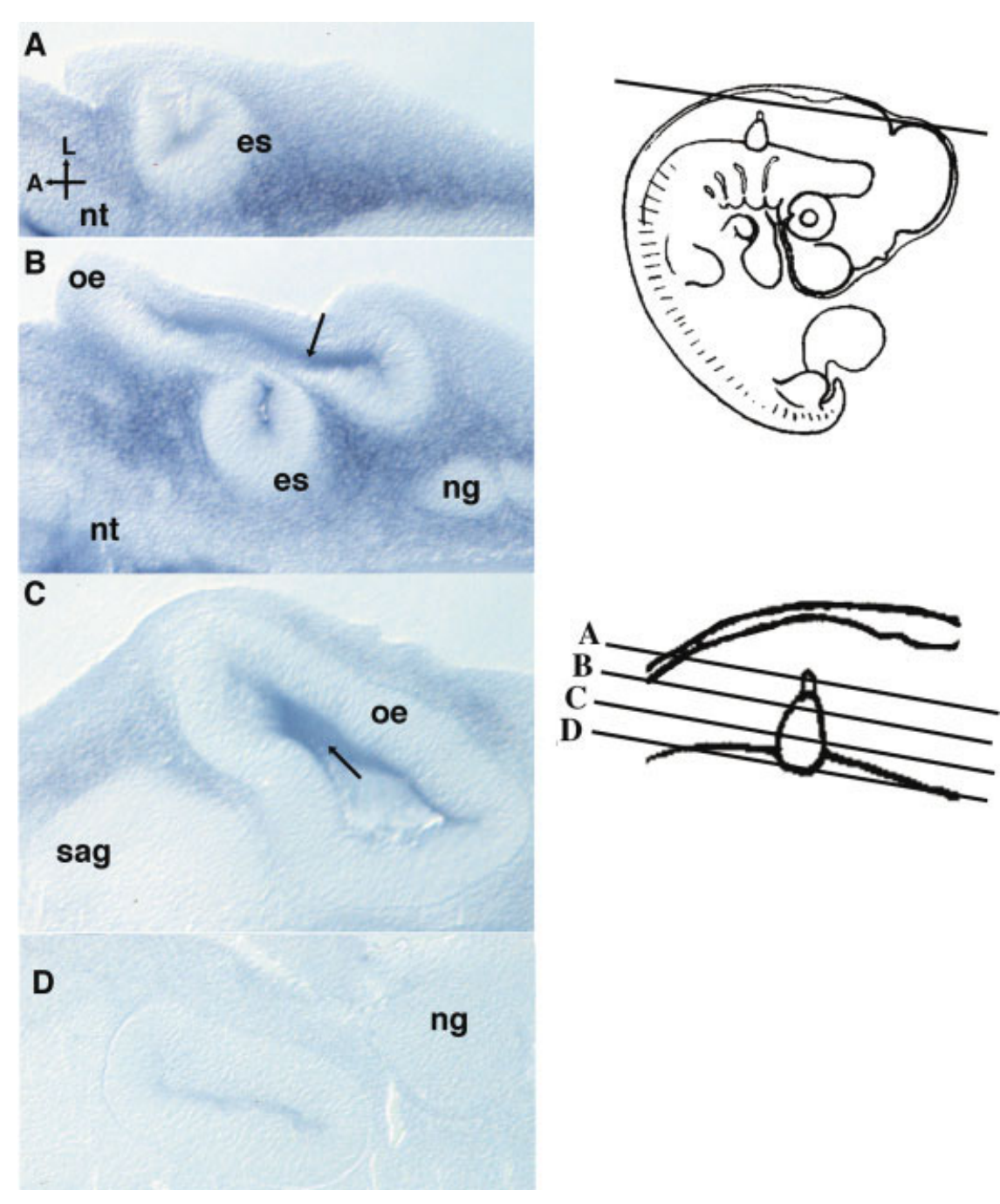

Fig. 7. ZIC2 expression in the periotic mesenchyme (POM) surrounding the otocyst of a stage 22 embryo sectioned as shown in the diagram to the right of the figures. Serial coronal sections through the otocyst were generated after whole-mount in situ hybridization. The level of each section is illustrated in the blow-up at the bottom right. Note that the POM surrounding the two dorsal-most sections of the otocyst $(A, B)$ is more heavily labeled than that of the most ventral part of the otocyst (D), and that lateral labeling (to the top of each section in $C$ and $D$ ) is more prominent than medial labeling (to the bottom). Neither the statoacoustic ganglion (sag) in $C$ nor the nodose ganglion (ng) in B and D expresses ZIC2. Orientation: A, anterior; L, lateral; es, endolymphatic sac; nt, neural tube; oe, otic epithelium. The arrows in $B$ and $C$ indicate nonspecific trapping, because this is a wholemount preparation of the embryo. Original magnifications, $\times 25$ in A-D.

expression in the sensory tissue of the scala media at this time, although a few epithelial lining cells of the scala tympani are labeled.

\section{CONCLUSIONS}

Four regions have been shown to be important in influencing the patterning of the developing inner ear: the hindbrain, the NC, the POM, and the notochord. We have found expression of $\mathrm{ZICl}$ and $\mathrm{ZIC} 2$ in the hindbrain, NC, and the POM during de- patterning of the inner ear through their expression by tissues that are critical for inner ear morphogenesis (Fritzsch et al., 1997) as well as in the auditory tissue itself.

We also propose that the $\mathrm{ZIC}$ genes may have a role in mediating neural competence of the otic epithelium. We have detected $\mathrm{ZICl}$ expression in the medial wall of the otocyst at stage 23 (Figs. 4C,D, 5B). At this stage, the statoacoustic ganglion (SAG) neurons, which do not express ZIC genes (Figs. 1G, 8B,C), are finding their way back to the otocyst to make contact with the presumptive sensory epithelium. The expression of $Z I C I$ in the epithelium and in the POM suggests that $\mathrm{ZICl}$ may be important in establishing these contacts. During stage 32, $\mathrm{ZICl}$ is still expressed in the mesenchymal cells surrounding the membranous labyrinth and within the crista ampulla (Figs. 4E, 6E). Persistence of $\mathrm{ZICl}$ expression in these regions also suggests that $\mathrm{ZICl}$ continues to play a role in innervation at and after stage 32. An important repair event that occurs in the chick basilar papilla after noise trauma is reinnervation of the hair cells (Ryals and Westbrook, 1994; Wang and Raphael, 1996). Studying the expression of ZIC genes relative to specific innervation events both in development and in regeneration should provide insight into the potential of reinnervation after noise trauma, ototoxicity or ageing that all affect the connections between the sensory hair cells and the nerve.

\section{EXPERIMENTAL PROCEDURES Isolation of Chick Probes for ZIC Genes}

The partial 233-bp chick cDNA for ZIC2 (amplicon KH163; GenBank no. AF188735) was cloned in a differential display (Liang and Pardee, 1992; Liang et al., 1993) experiment designed to identify genes that are differentially expressed in the chick cochlea after noise overstimulation (acoustic trauma) and during regeneration of the auditory epithelium (Gong et al., 1996). This CDNA sequence had high identity to the 3'-UTRs of mouse, Xenopus, and ze- 
brafish ZIC2 CDNAs, suggesting that we had cloned the chick ZIC2 CDNA. The other ZIC family members, including chick $\mathrm{ZICl}$ and $\mathrm{ZIC} 3$ probes, therefore, were subse- quently isolated by two different strategies. First, we aligned the amino acid sequences of all known ZIC family members to identify conserved regions. ZICl was cloned by
PCR of a chick cochlear cDNA library with primers to the conserved zinc finger domain: 5'-ATTCATGGACCTTCATGTG-3' and 5'-AAAACTTTCAGCACCATGC-3'. PCR prod-

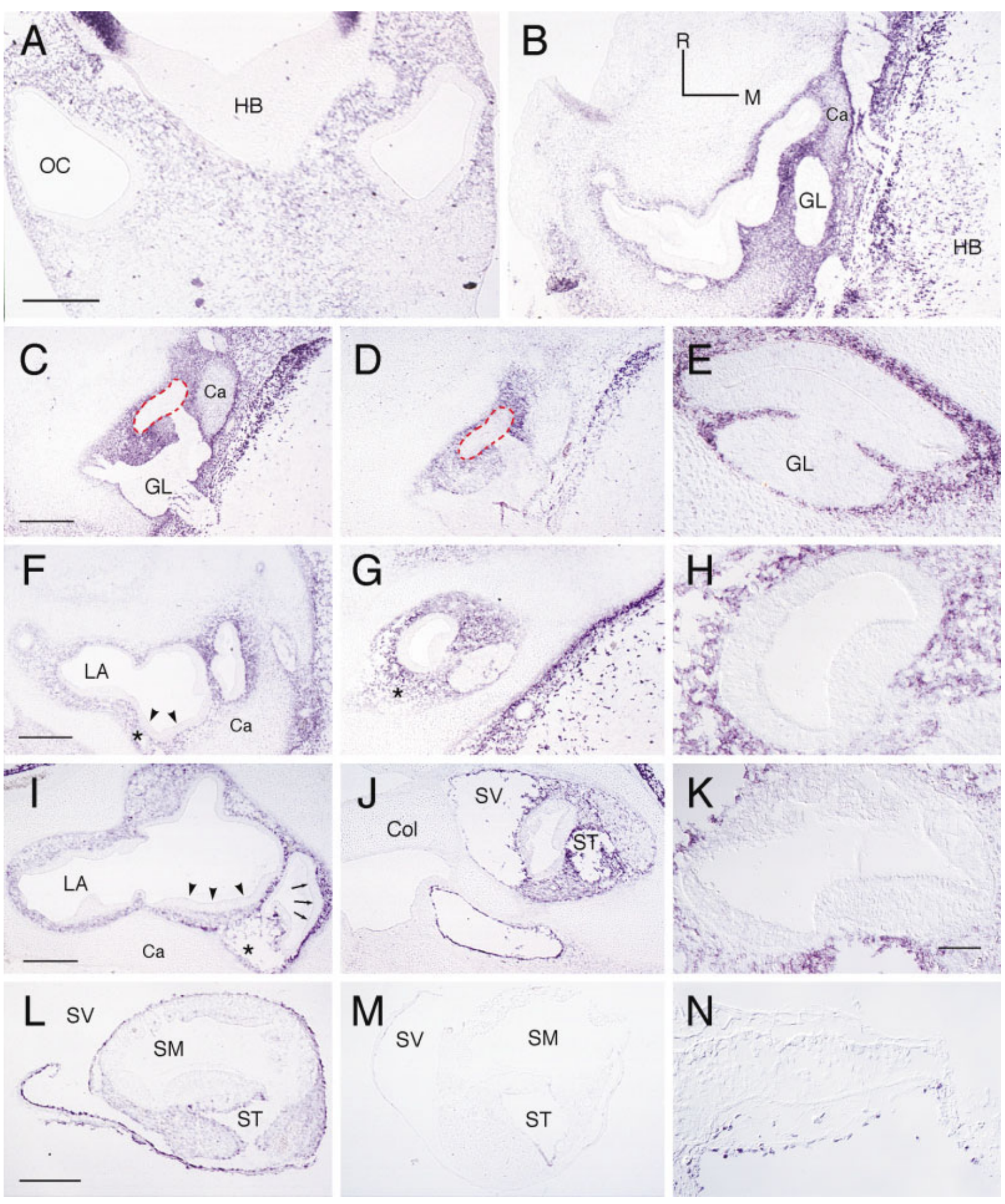

Fig. 8. 
ucts were cloned into PGEM-T Easy and sequenced. All eight clones tested were $\mathrm{ZICl}$. To isolate a ZIC3 probe, we aligned the CDNA sequences of all known ZIC3 family members and identified conserved regions specific for ZIC 3. The following primers (5'-ACGAATGGTACGTCTGAG-3' and 5'-TTCACAAGTGTAATGCTTG-3') were used for reverse transcriptase-polymerase chain reaction (RT-PCR) on day 10 chick embryo RNA. The PCR product was subcloned as for $\mathrm{ZICl}$. The sequence data reported in this study have been submitted to GenBank and have received the accession nos. AF188733 (human partial CDNA for ZIC2), AF188734 (chick ZIC1; KH509), AF188735 (chick ZIC2 amplicon; $\mathrm{KH} 163$ ), and AFI88736 (chick ZIC3; $\mathrm{KH} 540$ ).

\section{In Situ Hybridization of Chick Embryos}

In situ hybridization procedures were used to determine spatial and temporal expression patterns of $\mathrm{ZICl}$, ZIC2, and ZIC3. The 450-bp chick ZICI and 500-bp chick ZIC3 clones isolated by PCR, and the 233-bp chick ZIC2 amplicon were linearized and used to generate digoxigenin (DIG) -labeled riboprobes. Both the $\mathrm{ZICl}$ and $\mathrm{ZIC} 3$ sense riboprobes were prepared by linearizing the plasmid with Ncol and transcribing with SP6 RNA polymerase, whereas the antisense riboprobes were prepared by linearizing the plasmid with Pstl and transcribing with $T 7$ RNA polymerase. Templates for ZIC2 sense and antisense riboprobes were linearized with NoHl and Ncol, and transcribed with T7 and SP6 RNA polymerases, respectively. Sense orientation riboprobes were used to confirm that there was no significant background labelling in in situ hybridization experiments.

Whole-mount in situ hybridization analysis on stage 10-24 embryos was performed essentially as described previously (Gerlach et al., 2000, Gerlach-Bank et al., 2002). Alkaline phosphatase detection times varied for each probe, usually with $Z I C 2$ and $Z I C 3$ requiring two to three times longer than incubation times for ZIC7. After the in situ procedure, embryos were cryoprotected in 30\% sucrose, embedded in OCT (Miles), cryosectioned at 14-16 $\mu \mathrm{m}$, and mounted onto superFrost slides (VWR) coated with gelatin. Tissues used to generate frozen sections were fixed overnight at $4^{\circ} \mathrm{C}$ in $4 \%$ paraformaldehyde in phosphatebuffered saline (PBS), then equilibrated in $30 \%$ sucrose in PBS for up to 1 week. The tissues were embedded in OCT, frozen, and cut on a Microm cryostat (Zeiss) at $8 \mu \mathrm{m}$. The sections were transferred onto SuperFrost Plus slides, air-dried for 2-6 hr at room temperature, and stored at $-80^{\circ} \mathrm{C}$. In situ hybridization with frozen sections was performed according to published procedures (Jensen and Wallace, 1997; Jensen, personal communication), except that the color reaction was performed in the presence of $10 \%$ polyvinyl alcohol to enhance the signal, as described by DeBlock and Debrouwer (1993).

\section{Double In Situ Hybridization With ZICl and Chordin}

For double-labeled in situ hybridization on frozen sections, ZICl riboprobe was synthesized as described, except that it was labeled with fluorescein and detected with an antifluorescein antibody (Roche). The Chordin riboprobe, provided by $\mathrm{K}$. Lee (Columbia University), was prepared by using a DIG-RNA labeling mix as previously described by Streit et al. (1998) and detected with an anti-DIG antibody (Roche). Both riboprobes were added to the hybridization solution and in situ hybridization was performed on frozen sections as described by Jensen and Wallace (Jensen and Wallace, 1997; Jensen, personal communication) with modifications described by Dong Liu, University of Oregon (personal communication). After incubation with the anti-fluorescein antibody, the tissue sections were rinsed in Tris buffered saline (TBS) and then incubated at $37^{\circ} \mathrm{C}$ in Fast Red (Sigma) until the desired labeling intensity for $\mathrm{ZICl}$ was obtained. The slides were rinsed in TBST for $5 \mathrm{~min}$, then fixed for $10 \mathrm{~min}$ in $10 \mathrm{mM} \mathrm{HCl}$. After two additional TBST washes, the slides were incubated overnight at $4^{\circ} \mathrm{C}$ with the anti-DIG antibody. The sections were subsequently rinsed in

Fig. 8. Expression of $\mathrm{ZICl}$ and $\mathrm{ZIC} 2$ at later stages of inner ear development. In situ hybridization of frozen sections of chicks from embryonic day (E) 4 through posthatch day 7 . Sections A-K are coronal; sections L-N are cross-sections of the basilar papilla, which was first dissected out. A: $\mathrm{E} 4 \mathrm{ZICl}$ expression is seen in the periotic mesenchyme between the otocysts (OC) and the hindbrain (HB). B: E7 coronal section through the developing inner ear at the level of the statoacoustic ganglion (GL) shows that $Z I C l$ is expressed in the condensing cartilage (Ca). Comparison of $Z I C l$ and ZIC2 expression patterns at E7 are seen in C: (ZICl) and D (ZIC2). Adjacent 10- $\mu \mathrm{m}$ sections through the hindbrain and developing basilar papilla (dotted outlines in C,D) were labeled with each probe at E7. Note that the ZICl and ZIC2 expression patterns overlap but that ZIC2 expression is lower than ZICl in all labelled tissues. E: ZICl expression at E7. Only cells lining the perilymphatic space expressed ZICl. GL is the statoacoustic ganglion. F: ZICl expression at E9 through the lateral ampulla. The arrowheads represent the macula utriculus. The macular saculus is the chamber to the right of the MU. *indicates the sensory epithelium. $\mathrm{ZICl}$ expression is seen only in the condensing cartilage and perichondrium immediately surrounding the inner ear. Low-power $(\times 10, G)$ and high-power $(40 \times, \mathrm{H})$ views of an E9 section through the basilar papilla probed with ZICl. No ZICl expression is found in the sensory tissue, seen in $\mathrm{H}$ enlarged from the area with the asterisk in $\mathrm{G}$ but is found only in the surrounding cartilage. I: An E12 section through the lateral ampulla (LA), the utricular macula (arrowheads), and the saccular macula (arrows). The sensory epithelium does not express ZICl. $\mathrm{J}$ : An E12 section through the basilar papilla (seen at high power in $\mathrm{K}$ in a section rotated 90 degrees clockwise) and through the scala vestibuli (SV) and the scala tympani (ST) labeled with a probe for ZICl. K: Note that the sensory tissue (seen at high power) does not express $\mathrm{ZICl}$. L: Higher magnification of $\mathrm{ZICl}$ expression surrounding the developing basilar papilla and ganglion at E16. The SV, scala media (SM), and ST are shown. However, there is no ZICl expression in the sensory tissue of the SM. Posthatch day 7 basilar papilla, showing the SV, SM, and ST at low power (M) and high power (N). No ZICl expression is seen in the sensory tissue. N: A few epithelial lining cells of the scala tympani are ZICl positive. R, rostral; $M$, medial. Scale bars = $100 \mu \mathrm{m}$ in $A$ (applies to $A, B$ ), in $C$ (applies to $C$-E), in $F$ (applies to F-H), in I (applies to I-K), in L (applies to L-N). 
the prestain solution used by Jensen and Wallace (1997) before incubation with NBT/BCIP (Roche) as in the single label in situ hybridization protocol.

\section{Immunohistochemistry}

Rabbit polyclonal antibody recognizing chick Pax2 protein was obtained from BabCO/Covance (Richmond, CA) and was used at a 1/100 or $1 / 200$ dilution followed by a goat anti-rabbit secondary antibody (Jackson Immunoresearch) at a $1 / 500$ dilution as in our previously published methods (Hutson et al., 1999).

\section{ACKNOWLEDGMENTS}

We thank Amanda Ellis for expert cryosectioning. Thanks to Drs. John Germiller (University of Michigan), Donna Fekete (Purdue University), Guy Richardson (University of Sussex), and Jennifer Stone (University of Washington) for helpful discussions and/or valuable comments on the manuscript. Thanks to Drs. Abbie Jensen and Dong Liu (University of Oregon) for helpful discussions and in situ hybridization protocols. M.R.H and L.G.-B. (K.F.B., mentor) were supported by fellowships from the Hearing and Chemical Senses Training Grant. S.-H. Oh was a scholar of the Korean Science and Engineering Foundation. K.F.B. and M.I.L. received funding from the National Institutes of Health and K.F.B received funding from the National Science Foundation.

\section{REFERENCES}

Aruga J, Yokota N, Hashimoto M, Furuichi T, Fukuda M, Mikoshiba K. 1994. A novel zinc finger protein, zic, is involved in neurogenesis, especially in the cell lineage of cerebellar granule cells. J Neurochem 63:1880-1890.

Aruga J, Nagai T, Tokuyama T, Hayashizaki Y, Okazaki Y, Chapman VM, Mikoshiba K. 1996. The mouse zic gene family, homologues of the Drosophila pairrule gene odd-paired. J Biol Chem 271: 1043-1047.

Aruga J, Minowa $\mathrm{O}$, Yaginuma H, Kuno J, Nagai T, Noda T, Mikoshiba K. 1998. Mouse Zicl is involved in cerebellar development. J Neurosci 18:284-293.

Bissonnette JP, Fekete DM. 1996. Standard atlas of the gross anatomy of the developing inner ear of the chicken. J Comp Neurol 368:620-630.

Brown SA, Warburton D, Brown LY, Yu C-Y, Roeder ER, Stengel-Rutkowski S, Hennekam RCM, Muenke M. 1998. Holoprosencephaly due to mutations in ZIC2, a homologue of Drosophila oddpaired. Nat Genet 20:180-183.

Chang W, Nunes FD, De Jesus-Escobar JM, Harland R, Wu DK. 1999. Ectopic noggin blocks sensory and nonsensory organ morphogenesis in the chicken inner ear. Dev Biol 216:369-381.

Cramer KS, Rosenberger MH, Frost DM, Cochran SL, Pasquale EB, Rubel EW. 2000. Developmental regulation of EphA4 expression in the chick auditory brainstem. J Comp Neurol 426:270-278.

DeBlock M, Debrouwer D. 1993. RNA-RNA in situ hybridization using digoxigeninlabeled probes: the use of high molecular-weight polyvinyl alcohol in the alkaline phosphatase indoxyl-nitroblue tetrazolium reaction. Anal Biochem 215:86-89.

Fekete DM. 1999. Development of the vertebrate ear: insights from knockouts and mutants. Trends Neurosci 22:263268.

Fritzsch B, Barald KF, Lomax MI. 1997. Early embryology of the vertebrate ear. In: Popper AN, Rubel E, Fay RR, editors. Handbook of the auditory system. New York: Springer-Verlag. p. 80-145.

Gerlach LM, Hutson MR, Germiller JA, Nguyen-Luu D, Barald KF. 2000. Addition of the BMP4 antagonist, noggin, disrupts avian inner ear development. Development 127:45-54.

Gerlach-Bank LM, Ellis AG, Noonen B, Barald KF. 2002. Cloning and expression of the chick DAN gene, an antagonist of the BMP4 family of growth factors. Dev Dyn 224:109-115.

Giudice G. 2001. Conserved cellular and molecular mechanisms in development. Cell Biol Int 25:1081-1090.

Gong T-WL, Hegeman AD, Shin JJ, Adler HJ, Raphael Y, Lomax MI. 1996. Identification of genes expressed after noise exposure in the chick basilar papilla. Hear Res 96:20-32.

Hamburger V, Hamilton HL. 1951. A series of normal stages in the development of the chick embryo. J Morphol 88:49-92.

Herbrand H, Guthrie S, Hadrys T, Hoffmann $\mathrm{S}$, Arnold $\mathrm{HH}$, Rinkwitz-Brandt $\mathrm{S}$, Bober E. 1998. Two regulatory genes, CNkx5-1 and CPax2, show different responses to local signals during otic placode and vesicle formation in the chick embryo. Development 125:645654.

Hutson MR, Lewis JE, Nguyen-Luu D, Lindberg KH, Barald KF. 1999. Expression of Pax-2 and patterning of chick inner ear. J Neurocytol 28:795-807.

Ivanova A, Yuasa S. 1998. Neuronal migration and differentiation in the development of the mouse dorsal cochlear nucleus. Developmental Neuroscience 20:495-511.

Jensen AM, Wallace VA. 1997. Expression of Sonic hedgehog and its putative role as a precursor cell mitogen in the developing mouse retina. Development 124:363-371.

Larrain J, Bachiller D, Lu B, Agius E, Piccolo S, De Robertis EM. 2000. BMP-binding modules in Chordin: a model for signalling regulation in the extracellular space. Development 127:821-830.

Liang P. Averboukh L, Pardee AB. 1993. Distribution and cloning of eukaryotic mRNAs by means of differential display: refinements and optimization. Nucleic Acids Res 21:3269-3275.

Liang P, Pardee AB. 1992. Differential display of eukaryotic messenger RNA by means of the polymerase chain reaction. Science 257:967-971.

Mizuseki K, Kishi M, Matsui M, Nakanishi S, Sasai Y. 1998. Xenopus Zic-related-1 and Sox-2, two factors induced by Chordin, have distinct activities in the initiation of neural induction. Development 125:579-587.

Nagai T, Aruga J, Takada S, Gunther T, Sporle R, Schughart K, Mikoshiba K. 1997. The expression of the mouse Zicl. Zic2, and Zic3 gene suggests an essential role for Zic genes in body pattern formation. Dev Biol 182:299-313.

Nagai T, Aruga J, Minowa O, Sugimoto T, Ohno Y, Noda T, Mikoshiba K. 2000. Zic2 regulates the kinetics of neurulation. Proc Natl Acad Sci U S A 97:1618-1623.

Nakata K, Nagai T, Aruga J, Mikoshiba K. 1997. Xenopus Zic3, a primary regulator both in neural and neural crest development. Proc Natl Acad Sci U S A 94: 11980-11985.

Nakata K, Nagai T, Aruga J, Mikoshiba K. 1998. Xenopus Zic family and its role in neural and neural crest development. Mech Dev 75:43-51.

Ryals BM, Westbrook EW. 1994. TEM analysis of neural terminals on autoradiographically identified cells. Hear Res 72 : 81-88.

Sasai Y, Lu B, Steinbeisser H, Geissert D, Gont LK, De Robertis EM. 1994. Xenopus Chordin: a novel dorsalizing factor activated by organizer-specific homeobox genes. Cell 79:779-790.

Stone JS, Rubel EW. 2000. Temproal, spatial, and morphologic features of hair cell regeneration in the avian basilar papilla. J Comp Neurol 417:1-16.

Streit A, Stern CD. 1999. Mesoderm patterning and somite formation during node regression: differential effects of Chordin and noggin. Mech Dev 85:8596.

Streit A, Lee KJ, Woo I, Roberts C, Jessell TM, Stern CD. 1998. Chordin regulates primitive streak development and the stability of induced neural cells, but is not sufficient for neural induction in the chick embryo. Development 125:507519.

Torres M, Giraldez F. 1998. The development of the vertebrate inner ear. Mech Dev 71:5-21.

Wang Y, Raphael Y. 1996. Re-innervation patterns of chick auditory sensory epithelium after acoustic overstimulation. Hear Res 97:11-18. 